\title{
Liquid biopsy in NSCLC: a new challenge in radiation therapy
}

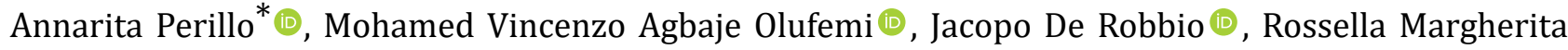



Department of Advanced Biomedical Sciences, University “Federico II” School of Medicine, Via Sergio Pansini 5, 80131 Napoli, Italy

*Correspondence: Annarita Perillo, Department of Advanced Biomedical Sciences, University “Federico II" School of Medicine, Sergio Pansini 5, 80131 Napoli, Italy. annaritap@live.it

Academic Editor: Carminia Maria Della Corte, Università degli studi della Campania, Italy

Received: May 24, 2020 Accepted: February 23, 2021 Published: April 30, 2021

Cite this article: Perillo A, Agbaje Olufemi MV, De Robbio J, Mancuso RM, Roscigno A, Tirozzi M, et al. Liquid biopsy in NSCLC: a new challenge in radiation therapy. Explor Target Antitumor Ther. 2021;2:156-73. https://doi.org/10.37349/etat.2021.00038

\begin{abstract}
Lung cancer is the most common cancer and the leading cause of cancer mortality worldwide. To date, tissue biopsy has been the gold standard for the diagnosis and the identification of specific molecular mutations, to guide choice of therapy. However, this procedure has several limitations. Liquid biopsy could represent a solution to the intrinsic limits of traditional biopsy. It can detect cancer markers such as circulating tumor DNA or RNA (ctDNA, ctRNA), and circulating tumor cells, in plasma, serum or other biological fluids. This procedure is minimally invasive, reproducible and can be used repeatedly. The main clinical applications of liquid biopsy in non-small cell lung cancer (NSCLC) patients are the early diagnosis, stratification of the risk of relapse, identification of mutations to guide application of targeted therapy and the evaluation of the minimum residual disease. In this review, the current role of liquid biopsy and associated markers in the management of NSCLC patients was analyzed, with emphasis on ctDNA and CTCs, and radiotherapy.
\end{abstract}

\section{Keywords}

Lung cancer, liquid biopsy, personalized therapy, radiotherapy, tissue biopsy

\section{Introduction}

Lung cancer is the most common cause of cancer incidence and mortality worldwide with 2.1 million newly diagnosed cases and 1.8 million deaths in 2018 [1]. According to the World Health Organization (WHO), lung cancers are divided in two main groups: non-small cell lung cancer (NSCLC; 85\% of cases) and small cell lung cancer (SCLC; $15 \%$ of cases). The predominant histological subtypes of NSCLC are adenocarcinoma (ADC; 40\% of cases) and squamous cell carcinoma (SqCC; $20 \%$ of cases) [2]. The majority of NSCLC are diagnosed when the disease is too extensive to be surgically removed. For these patients, chemotherapy, radiotherapy (RT) and targeted drug therapy are the standard of care. The gold standard for stage I and II NSCLC is surgery, possibly followed by adjuvant cisplatin-based chemotherapy if histological examination shows risk factors. For patients with contraindications or who are unfit for surgery, the first choice is RT as stereotactic body irradiation (SBRT) for stage I-II N0 or as concurrent definitive chemoradiotherapy for N+. Stage III 
is a heterogeneous group: potentially resectable tumors (stage IIIa) should be treated with neoadjuvant chemotherapy followed by surgery and adjuvant RT whereas unresectable tumors (some stage IIIa and all stage IIIb-IIIc) should be treated with concurrent definitive chemoradiotherapy followed by durvalumab, an immune checkpoint inhibitor that has been shown to improve both progression free survival (PFS) and overall survival (PACIFIC trial). In the metastatic setting, several factors such as histology, age, performance statistics, comorbidities and molecular pathway alterations should be considered in the selection of the best treatment strategies, which may include chemotherapy, immunotherapy and targeted drug therapy. Therefore, following morphological diagnosis, it is very important to evaluate predictive biomarkers of therapy. Two testing streams have evolved, one for the detection of targetable oncogenic alterations and the other for immune-oncology related biomarkers.

Diagnosis in NSCLC patients is based on tissue biopsy. Today, it must determine not only the histological subtypes (NSCLC vs. SCLC or SqCC vs. ADC) but also the potential presence of specific molecular alterations. However, this procedure has several limitations. Patient comorbidities and/or small nodules can make obtaining biopsies difficult and risky. As reported by Overman et al. [3], the complication rate for intrathoracic solid organ biopsies was $17.1 \%$ (36 of 211 biopsies). In some cases, the samples obtained are small and the content of neoplastic cells is insufficient to identify both the histotype and molecular alterations. In others cases, the samples obtained may be completely free of neoplastic cells [4]. In addition, as demonstrated in the study of Gerlinger et al. [5], lung cancer is characterized by a remarkable level of inter-tumor and intratumor heterogeneity. This often makes the tissue biopsy unrepresentative of the complete genetic makeup of the neoplasia. The procedure is expensive and not easily repeatable, and, for this reason, cannot be used to evaluate the temporal evolution of the disease or response to treatments. The identification of new mutations or genetic modifications is necessary to establish the most appropriate therapy for NSCLC patients, but it is evident that serial tissue biopsies are not the most suitable tools for this purpose.

In these situations, liquid biopsy could be a turning point for patient management. It can detect cancer markers such as circulating tumor DNA or RNA (ctDNA, ctRNA), and circulating tumor cells (CTCs), in plasma, serum or other biological fluids. Liquid biopsy makes it possible to access material derived from multiple tumour deposits through a procedure which is minimally invasive and repeatable. It has various potential clinical applications, such as early detection of tumor, identification of mutations for targeted therapy, evaluation of response to treatment, early identification of resistance and rapid detection of relapses.

In this review, we will analyze the current role of liquid biopsy in the management of NSCLC. The primary focus of the paper is on liquid biopsy and associated markers, especially ctDNA and CTCs, and their potential influence on RT.

\section{ctDNA and RNA}

ctDNA is the fraction of cell-free DNA that derives from lysis/necrosis of tumor cells, from tumor destruction by macrophages or by direct secretion [6]. Although circulating cell-free DNA (cfDNA) includes all DNA fragments present in biological fluids, including those of neoplastic origin, it mainly derives from nonmalignant hematopoietic cells. cfDNA was first identified in the blood by Mandel and Metais in 1948 [7]. ctDNA levels can vary enormously over time and between patients [8] and are influenced by tumor-related factors such as volume (stage TNM) and rate of proliferation, but also by factors affecting the systemic circulation. In patients with NSCLC the ctDNA level is usually $0.02-3.2 \%$ of cfDNA [9]. cfDNA is rapidly eliminated from the blood (the half-life of these fragments typically varies from minutes to $1-2 \mathrm{~h}$ ), and ctDNA can therefore provide real-time information on the tumor [10]. cfDNA usually has a size of 166 base pairs. This is similar to the nucleosomal fragments released during apoptosis, which could represent the primary source of cfDNA [11]. The size of ctDNA has long been debated and recent studies suggest that ctDNA is shorter than cfDNA [12-13]. ctDNA carries many of the molecular aberrations present in the tumor (such as single-nucleotide mutations [14] and methylation changes [15]) and this distinguishes it from cfDNA and permits its use as an oncological marker. It allows identification of mutations in various genes, such as EGFR, Kirsten rat sarcoma viral oncogene homolog (KRAS), BRAF, etc. The best source for ctDNA research is plasma since the presence of coagulation inhibitors prevents the lysis of white blood cells and therefore 
dilution of ctDNA by cfDNA [16]. The sample is typically acquired from peripheral veins into an tube and in order to minimize sample alteration, the time between blood sample collection, plasma isolation, ctDNA centrifugation and ctDNA extraction should be reduced as much as possible [17]. One solution to minimize the time is to perform all the phases of the analysis in the same laboratory or, alternatively, to use special tubes that prevent the release of cfDNA and the degradation of ctDNA (PAXgene Blood DNA tubes or Cell-Free DNA BCT tubes) $[18,19]$. Once collected, the blood is filtered or subjected to low and high speed centrifugation cycles [20].

After extraction, ctDNA profiling can be based on analysis of single mutations [generally through polymerase chain reaction (PCR)] or more broadly, using next generation sequencing (NGS) [21]. For PCR, quantitative PCR (qPCR) analysis is based on quantitation of the intensity of fluorescent light emitted by the probes in every PCR cycle, while droplet digital PCR (ddPCR) separates the sample into droplets containing either 1 or 0 molecules of DNA for subsequent amplification. Similar to ddPCR, BEAMing is a digital PCR method which uses water droplets in an oil emulsion as reaction vessels containing a mixture of template, primers and PCR reagents, and magnetic beads. NGS can be performed using several platforms, such as Illumina (San Diego, California) or IonTorrent (ThermoFisher Scientific, Waltham, Massachusetts), that sequence nucleic acids through a large number of parallel reads and their subsequent alignment to a genomic reference standard. This technology allows to analyze whole transcriptomes or a small group of RNAs. In contrast to PCR, NGS can detect rare and previously uncharacterized alterations and is particularly useful for detection of deleterious mutations which can accumulate in diverse positions within some tumor suppressor genes. Moreover, costs and analytical time associated with NGS have reduced remarkably.

As well as ctDNA, ctRNA is the fraction of circulating cell free RNA derived from tumor cells and it is detectable in the fluids of cancer patients. Compared with ctDNA, ctRNA is more unstable and it degrades very quickly. Its half-life can be increased by association with proteins or proteolipid complexes. In the context of ctRNA, miRNAs represent a promising biomarker for NSCLC. miRNAs are small noncoding RNAs (21-25 nucleotides) which modify target gene expression post-transcriptionally via inhibition on mRNA translation and induction of mRNA degradation. miRNAs have been associated with the main hallmarks of NSCLC, including sustaining proliferative signaling (miR-7, miR-30, miR-34), evading growth suppressors (miR-641, miR-660) and activating invasion (miR-200) [22]. Recently, a panel of miRNAs has been used for diagnosis and prognostication in lung cancer $[23,24]$.

\section{CTCs}

CTCs are cancer cells released from solid tumors into the blood circulation and were first observed in 1,869 by Ashworth [25]. CTCs are extremely rare (1 to 10 cells per $10 \mathrm{~mL}$ of blood) and have a half-life shorter than ctDNA (6-10 min for clusters vs. 25-30 min for single cells) [26]. Despite their short lifespan, these cells may contribute to metastasis and neoangiogenesis [27]. Several studies have shown that CTCs are an unfavorable prognostic factor in breast [28], prostate [29] and colorectal cancers [30]. Immunomagnetic separation is generally the most commonly used method for isolating CTCs. In this case, CTCs are positively enriched through the use of capture agent-labeled magnetic beads which bind to cell-surface markers [31, 32]. CellSearch assay [33], the only platform approved by the Food and Drug Administration (FDA) for CTC enumeration is based precisely on this mechanism. Conversely, it is possible to negatively enrich the CTCs by using anti-CD45 to deplete leukocytes [34]. Genomic analysis of CTCs can be performed by PCR, NGS and fluorescent in situ hybridization (FISH) as well as ctDNA [35].

The main advantages and limitations of ctDNA, ctRNA and CTCs detection techniques are shown in Table 1.

\section{Clinical applications of liquid biopsy in NSCLC}

The main clinical applications of the liquid biopsy in NSCLC patients (Figure 1) are early diagnosis, stratification of risk of relapse, identification of gene mutations, and evaluation of the minimum residual disease (MRD). 
Table 1. Pros and cons of analysis methods used in liquid biopsies

\begin{tabular}{|c|c|c|}
\hline Analysis method & Pros & Cons \\
\hline \multirow[t]{2}{*}{ qPCR } & Good sensitivity and specificity & Limited to HTS and discovery studies \\
\hline & Widely available & \\
\hline ddPCR & High sensitivity & Expensive \\
\hline BEAming & $\begin{array}{l}\text { Detection of ctDNA with very low mutant allele } \\
\text { frequency }\end{array}$ & Expensive \\
\hline \multirow[t]{2}{*}{ NGS } & High sensitivity & Bioinformatics support required \\
\hline & Identification of several miRNA variants & No automation \\
\hline \multirow[t]{3}{*}{ Whole-genome sequencing } & Wide application & Expensive \\
\hline & & Time-consuming \\
\hline & & Bioinformatics support required \\
\hline \multirow[t]{3}{*}{ CellSearch } & FDA approved & Expensive \\
\hline & Good sensitivity and reproducibility & Limited to CTCs with high EpCAM levels \\
\hline & & False positives due to inflammation \\
\hline
\end{tabular}

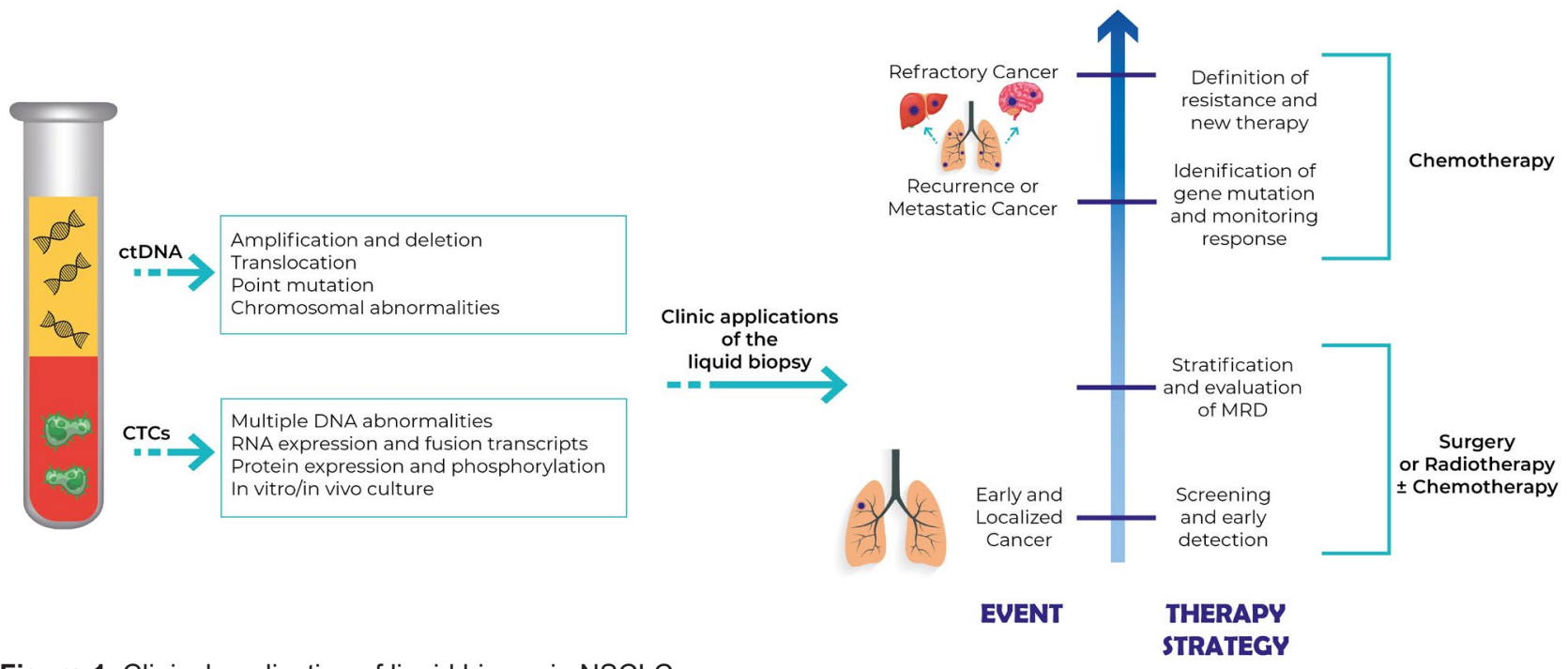

Figure 1. Clinical application of liquid biopsy in NSCLC

\section{Liquid biopsy and early diagnosis in NSCLC}

The rapid evolution and the lack of specific early symptoms are the basis of the late diagnosis of NSCLC and $75 \%$ of patients present with advanced stage disease at diagnosis (stage III/IV) [36]. This late diagnosis associated with reduced survival and poor prognosis as patient survival at 5 years decreases dramatically from $55.1 \%$ in stage I to $4.2 \%$ in stage IV. Early detection of lung cancer would radically change the survival of these patients. Unlike cancers such as breast, cervical and colorectal, there are no cancer screening programs for lung cancer; only in some countries is low-dose CT (LDCT) available for higher-risk individuals (55-74 years, $\geq 30$ pack years, smoked within 15 years). The National Lung Screening Trial (NLST) study was the first to demonstrate how LDCT is associated with a $20 \%$ reduction in lung cancer mortality compared to chest X-ray [37]. Despite the advantages of LDCT, it should be remembered that this form of screening is associated with a high rate of false positives (81\%) [38] and overdiagnosis of indolent tumors such as lepidic ADCs. In the NELSON trial [39] the false positive rate was reduced to $59.4 \%$ due to "nodule management protocol" (evaluation of dimension and volume doubling time), while the cumulative lung cancer detection rate was substantially higher. In addition to this imaging, liquid biopsy may be another possible tool for lung cancer screening.

In 2014 Newman et al. [40], found ctDNA in 100\% of stage II-IV and 50\% of stage I NSCLC patients using the Cancer Personalized Profiling by deep Sequencing (CAPP-Seq). This study has certainly underlined the possible role of ctDNA for the evaluation of the response to treatment and the level of MRD due to the 
link between ctDNA level and stage, but also its possible role in early diagnosis. A recent test combining the evaluation of a protein panel with mutations in the ctDNA (CancerSEEK) demonstrated a specificity $>99 \%$ and a sensitivity of 59\% in 104 patients with lung cancer stage I-III [41].

Another possibility for the early diagnosis of lung cancer, offered by ctDNA, is based on the search for hypermethylation of $\mathrm{CpG}$ islands in the promoter regions of tumor-suppressor genes. In a Chinese study [42], the methylation frequency of 9 genes was statistically higher in the tumor tissue of subjects with NSCLC than in healthy tissue and these nine genes also showed a significantly higher frequency of tumor-specific hypermethylation in NSCLC plasma, as compared with the plasmsa fromcancer-free individuals. A smaller panel of 5 genes also showed a sensitivity and specificity of $83.64 \%$ and $74 \%$ respectively for cancer diagnosis. The genes in the panel were: APC (involved in cell signaling), CDH13 (involved in cell adhesion), KLK10 (member of the human tissue kallikrein family of secreted serine proteases), DLEC1 (involved in cell proliferation/differentiation) and RASSF1A (involved in cell-cycle control). Except KLK10, all other genes were already known to have a role in lung cancer. The methylation status was detected using methylationspecific PCR. In Wielscher et al. [43], the methylation status of cfDNA was used as screening in subjects with lung cancer, interstitial lung disease (ILD), chronic obstructive pulmonary disease (COPD), and healthy controls. The test had $88 \%$ sensitivity and $90 \%$ specificity vs. the controls, and $88 \%$ vs. the ILD and COPD. In another study, the methylation of 3 genes was used to evaluate subjects with suspected lung nodule of these 150 had a stage I-II NSCLC and 60 were controls, the test had a sensitivity of $93 \%$ and a specificity of $62 \%$ [44]. More recently, Liang et al. [45], evaluated the role of the methylation profiling of cfDNA as a method for early detection of NSCLC and demonstrated that their method was a valid tool for differentiation between neoplastic lesions and benign pulmonary nodules.

Unlike other tumors, the detection of CTCs is complex in NSCLC. In Krebs et al. [46], CTCs were detected only in $23 \%$ patients with NSCLC stage III-IV using the CellSearch system. The combined use of different detection techniques (CellSearch and Isolation by size of epithelial tumor cells) increased detection rates [47]. Ilie et al. [48], suggested a role for CTCs in the early detection of lung cancer in subjects with COPD. In their study, 168 subjects with COPD were evaluated with annual LDCT and, in 5 of these patients, CTCs were found 1 to 4 years before the CT finding of lung nodules then recognized as tumors. CTCs were not found in the control group. In Yu et al. [49], the efficacy of folate receptor (FR)-based CTC detection method in the diagnosis of NSCLC was evaluated. This method had a sensitivity of $73.2 \%$ and a specificity of $63.2 \%$ (also in stage I). The authors stressed that their method showed greater diagnostic efficacy than other clinical markers [neuron-specific enolase (NSE), carcinoembryonic antigen (CEA), CA125, cyfra21-1, and squamous cell carcinoma antigen (SCC Ag)].

Similar to ctDNA, miRNAs can be used for early detection of NSCLC. In Ma et al. [50], analysis of two miRNAs (miRs-19b-3p and -29b-3p) identified NSCLC with a sensitivity of $72.62 \%$ and a specificity of $82.61 \%$. These values increased in the identification of the SqCC ( $80 \%$ sensitivity and $89.86 \%$ specificity). Moreover, the panel formed by miR-193b, miR-301, miR-141 and miR-200b [51] showed great accuracy in discriminating patients with NSCLC (AUC 0.985). In the study of Dou et al. [52], 480 patients were analyzed (120 NSCLC and 360 healthy controls). The authors showed that the expression of let-7c and miR152 in plasma were downregulated in NSCLC patients. These biomarkers also correlated with histological classifications, differentiation status, lymph node metastasis and stage classifications so they could represent a tool for identify patients with NSCLC.

In the future, combined use of LDCT and introduction of liquid biopsy into clinical practice may allow earlier diagnosis by increasing the number of patients susceptible to local radical treatments such as surgery and SBRT [53]. However, to date, the lack of appropriate technologies and standardized protocols does not make it possible to use liquid biopsy in daily clinical practice.

\section{Liquid biopsy and stratification of the risk of relapse in NSCLC}

To date, modern RT techniques such as intensity modulation radiotherapy (IMRT) are highly conformational so this system can deliver high doses to the target volume while preserving the surrounding healthy tissues [54]. Different biological parameters influence cancer cells' sensibility to damage induced by ionizing 
radiation (IR), such as hypoxia, reoxygenation and cellular repopulation capacity, damage repair ability, cellular redistribution of cells in the different phases of cell cycle, as well as the intrinsic radiosensitivity of each type of tumor $[55,56]$. These radiobiological features explain both the heterogeneity of the response to the RT of the different tumors or the same tumor in different patients, and individual variety in the development of adverse events by RT. The tumor genome evolves dynamically over time and accumulates genetic alterations in different subclones [57]. This feature results in an intratumoral heterogeneity both spatially (i.e. between the primary tumor and the metastatic sites or, even, within the tumor nodule itself) and temporal (i.e. the bio-molecular characteristics of the tumor may change over time). Blood ctDNA levels change continuously according to tumor size, cancer stage, treatment status, etc. In this way, liquid biopsy could be a useful tool to quantify ctDNA serially to obtain a dynamic picture of the molecular evolution of the disease. It could play a very promising role in RT to guide the decision-making process for the treatment strategies and allowing monitoring of the response to the treatment, resistance development and disease progression.

ctDNA before RT: radiosensitivity and radioresistance

Tumor genetic evaluation has become increasingly important in clinical practice, in particular to guide targeted therapies [58], but also has a potentially crucial role in RT. The discovery of prognostic and predictive biomarkers could aid stratification of patients based on the probability of responding to treatment or development of adverse events $[59,60]$. In the future, such stratification will help in the choice between intensification or de-escalation dose treatments [61] and, therefore, to the development of increasingly personalized RT.

Quantitative analysis of pre-treatment ctDNA concentration in plasma is useful in the prognostic evaluation, in the follow-up [62-65] and in the identification of specific genetic alterations associated with radiosensitisation. Pre-RT genetic analysis could be fundamental in the stratification of patient as potential responders and non-responders. The mutations of several genes in different tumor settings such as NRF2 (nuclear factor erythroid 2-related factor 2), KEAP1 (kelch-like ECH-associated protein 1), and $K R A S$, have been identified as radiosensitising or radioresistant factors [66, 67]. One of the most promising biomarkers is the mutation of the KEAP1-NRF2 pathway which has been found in several cancers including NSCLC [68-72] and, in particular, in about one third of patients with SCLC. To date, the KEAP1-NRF2 pathway is known as a defense mechanism that cells activate in response to oxidative stress and to damage produced by toxins and xenobiotics with which they come into contact. This pathway is altered in cancer cells and this contributes to the development and progression of lung cancer $[73,74]$, and resistance to RT. In normal cells, KEAP1 binds NRF2 and targets it for proteasomal degradation [75, 76]. However, in response to oxidative stress, NRF2 is released from KEAP1, allowing it to accumulation and movs to the nucleus where it promotes the transcription of genes involved in defense against reactive oxygen species (ROS). Jeong et al. [67] found that the deletion of the KEAP1 promoter results in constitutive activation of NRF2 which favors tumor aggressiveness and metastasis, and upregulates ROS scavenging capacity and therefore resistance to oxidative stress. It is known that IR kills cancer cells due to double-stranded DNA damage induced by ROS [77] and the KEAP1 deletion (and consequent activation of NRF2) therefore leads to ROS suppression. This event promotes radioresistance and limits the effectiveness of RT in NSCLC patients. Furthermore, KEAP1/NRF2 mutations in patients with NSCLC seems to correlate with an increase in local recurrence after RT [67] suggesting that KEAP1/NRF2 status is predictive for local recurrence after RT in patients with lung cancer. This study shows that the identification of KEAP1/NRF2 mutations status, assessed with the analysis of ctDNA, is an important biomarker of effectiveness of treatment [67]. It could have a predictive value in RT outcome of NSCLC patients.

Even the methylation status of the tumor could be a valid biomarker assessed with ctDNA analysis. According to several studies [78-83], methylation is correlated with the aggressiveness of the tumor and it promotes tumor progression by silencing genes involved in regulating tumor growth and metastatic potential. It has been shown that the reduction of ten-eleven translocation (TET), enzyme activity induced by tumor hypoxia, causes DNA hypermethylation [84] and hypoxia is known to be one of the main biological factors of radioresistance. Therefore, variations within the tumor microenvironment can influence the response to RT and their identification through ctDNA could be a starting point for future studies. 
The role of transforming growth factor $\beta$ (TGF- $\beta$ ) is important as it is activated in many solid tumors and is associated with malignant progression through different interactions in the tumor cells and in the surrounding microenvironment. TGF- $\beta$ plays an important role as a tumor suppressor until invasive cancer development occurs, when a "switch" is activated, leading TGF- $\beta$ signaling to promote the proliferation and invasion of cancer cells. IR can activate the TGF- $\beta$ pathway with cross-talk activation with cox-2 [85] and TGF- $\beta$ also plays an important role in the response of normal tissues to RT, especially in the lung [86]. ROS produced by IR promote the secretion of TGF- $\beta$ [87] and its transcription increases within hours after irradiation [88]. Anti-TGF- $\beta$-antibodies have been shown to reduce inflammation, activation and expression of TGF- $\beta$ and radiation-induced fibrosis [89] so inhibition of the TGF- $\beta$ pathway could be very important for reducing damage, induced by irradiation, especially in lung. In addition, as demonstrated in several studies [90, 91], TGF- $\beta$ promotes the survival of cancer cells after irradiation and its inhibition leads to radiosensitization in different types of cancer. Inhibition of TGF- $\beta$ has a double role: protection of normal tissues and radiosensitization in the tumors. For this reason, studies that evaluate the inhibition of this pathway during or after treatment (conventional chemoradiation, radiosurgery) in NSCLC are very interesting.

Two important cellular targets in NSCLC are EGFR and K-RAS, whose mutations are mutually exclusive.

Usually, EGFR is mutated in ADC that most frequently occurs in young, Asian, female and non-smoking patients. EGFR is part of the family of HERs. It contains a C-terminus intracellular region that possesses the kinase activity and an $\mathrm{N}$-terminus extracellular ligand-binding site; it is implicated in epithelial tissues maintenance and growth. Increased EGFR expression has been associated with radioresistance [92]. $K$ - $R A S$ is one of the most commonly mutated proto-oncogenes in various tumors, including in lung cancer in smokers. The activation of $K$-RAS seems to increase radiosensitivity in cell lines [93]. The role of EGFR and $K-R A S$ in RT is still subject of numerous studies while their primary role is clear in the target therapy of lung cancer patients.

ctDNA during RT: assessment of response to the treatment and prediction of toxicity

The half-life of ctDNA in the blood is very short so it provides a near "real-time" indicator of tumor kinetics [94-96]. The longitudinal determination of the ctDNA may reflect the rapid death of the cancer cells. Its determination during RT could be indicate the cytotoxic action of IR and thus be a marker of effectiveness and response to RT [97-99].

Another clinical application of ctDNA is the early determination of treatment resistance. It has long been known that there is (in addition to the inter-tumor heterogeneity in the response to radiation [100-102]) intra-tumor heterogeneity [103-106] and this could contribute to radioresistance. Radioresistancepromoting mutations seem to involve genes implicated in cell survival, tumor suppression, ROS, cell cycle checkpoints, etc [107]. The identification of resistant clones with ctDNA analysis could be very useful for dose modifications during RT.

The normal tissues surrounding the tumor inevitably receive a small dose of radiation which can cause the onset of acute (during treatment and in the first weeks after RT) or late (several months or years after treatment) toxicity. Several studies have evaluated the influence of genetic markers, especially single nucleotide polymorphisms (SNPs), on radiological toxicity [108]. These studies focused mainly on the genes involved in repairing DNA damage (e.g., BRCA1, BRCA2, and ATM), cytokines (e.g., TNF and TGFB1) and antioxidant enzymes (e.g., superoxide dismutase) [109-111], and demonstrated a potential association between SNPs in these genes and RT toxicity [112-114]. Further studies are needed to evaluate the correlation between genetic alterations and adverse events from RT in order to develop a personalized RT.

\section{Liquid biopsy and identification of gene mutation in NSCLC}

Liquid biopsy can be used to detect mutations of genes involved in development of cancer, progression and response to therapy.

One of the most important markers in NSCLC is EGFR. This gene may exhibit different mutations which can influence sensitivity or resistance to tyrosine kinase inhibitors (TKIs) such as gefintib, erlotinib and afatinib. The most common mutations are sensitizing and typically consist of deletions in exon 19 and points mutation in exon 21 (L858R) [115]. The first occurs in 90\% of cases while the second is less common; these 
mutations are associated with better response rates and longer progression-free survival. In contrast, most of the exon 20 insertions are usually associated with TKIs resistance. In addition, some patients with mutated EGFR can acquire resistance to first- (erlotinib, gefitinib) or second- (afatinib) generation TKIs through the T790M mutation [116]. Prediction of the treatment outcome requires evaluation of the levels of both sensitizing and de-sensitizing mutations. The FDA approved the detection of these mutations using liquid biopsy. In a recent study, Mayo-de-las-Casas et al. [117], showed the absence of differences between the PFS of mutated EGFR patients detected by liquid biopsy compared to those detected by tissue biopsy after therapy with TKIs. In addition, Buder et al. [118], have highlighted how detection of T790M mutations in cfDNA can be a valuable tool to identify patients with resistance to osimertinib. It should also be remembered that detection of mutated EGFR in the plasma correlates with cancer responses to treatments measured with response evaluation criteria in solid tumors (RECIST) criteria [119]. Another great possibility provided by liquid biopsy is the early evidence of disease progression. Monitoring for increases in abundance of EGFR mutations detected in liquid biopsies (for example, an increase of $20 \%$ compared to the lowest value achieved during treatment) could predict progression 8 months before it was objectively detectable [120].

Another gene of particular importance in the NSCLC is anaplastic lymphoma kinase $(A L K)$ which is translocated in 3-7\% of lung cancer cases [121]. The most common fusion partner of $A L K$ is echinoderm microtubule-associated protein-like 4 (EML4). Translocation can be detected by liquid biopsy using PCR or NGS and it can be helpful in guiding an optimal choice of a TKI. Recent evidence suggests that L1196M and S1206Y mutations of $A L K$ cause resistance to crizotinib but not to ceritinib while I1171T and V1180L mutations cause resistance to alectinib and crizotinib, but not to ceritinib [122,123]. Over time, several other fusion partners for which the clinical implication is not yet known, have been identified. The use of liquid biopsy may not be the most effective method to identify these new translocations.

Similar to $A L K$ mutations (although less frequent; 1-3\%), ROS-1 mutations are also associated with altered sensitivity to TKIs. ROS-1 mutations are found mainly in never smokers, ADC and younger patients. Following the results of Shaw et al. [124], crizotinib was approved by the FDA for treatment of advanced ROS1-rearranged NSCLC. ROS-1 rearrangements can also promote resistance to crizotinib through acquisition of additional mutations in the kinase domain or by "off target" alterations in parallel pathways. The G2032R mutation is the most frequent ROS-1 resistance mutation and to date, only cabozantinib has shown activity against tumor clones carrying this mutation. Unfortunately, its toxicity limits its use in clinical practice [125].

To date, there are no specific drugs targeted against ROS-1 mutations, as in the case of KRAS. However, assessment is relevant as it is associated with a worse prognosis and reduced responsiveness to EGFRtargeted TKIs [126].

Analysis of CTCs has great, but still unrealized, promise for guiding therapy decisions. To date, the only FDA-approved method for their analysis is ineffective in patients with NSCLC. In a recent study, the use of an alternative method for CTC detection has been shown to be more effective. The study, in addition to evaluating this new method, also analyzed the expression levels of PD-L1 ${ }^{+}$, highlighting how their increase in the CTCs is associated with a higher probability of resistance to PD-1/PD-L1 inhibitors [127].

\section{Liquid biopsy and the evaluation of the MRD in NSCLC}

To date, the follow-up of NSCLC patients is dependent on imaging which, despite improvements, it appears unable to identify microscopic disease. Another limitation of radiological methods is the difficulty of distinguishing between residual/recurrence disease and RT alterations. Today, RT is increasingly used in lung cancer thanks to the improvements of stereotactic and hypofractional methods, so it is becoming increasingly urgent to add to radiology other more sensitive methods in the search for residual disease. One of the most interesting potential uses of analysis of ctDNA is its possible role in detection of minimal tumor residual disease. MRD usually shows the presence of a small number of malignant cells in a patient otherwise considered in clinical remission. Since cancer often relapses after or during treatment, disease monitoring and treatment evaluation are important for clinicians to determine other treatment protocols. Preliminary data on the clinical utility of ctDNA in MRD detection is promising as demonstrated in several studies. 
Diehl et al. [128], analysed a cohort of patients with surgically-treated colorectal cancer. They monitored ctDNA and CEA levels after tumor resection and saw that ctDNA detection was strongly correlated to an increased rate of relapse (PFS at 3 years was $90 \%$ in patients with undetectable levels of ctDNA vs. 0\% in patients with detectable ctDNA). In addition, Bettegowda et al. [129], showed that the detection rates of ctDNA among the patients with cancer stage I, II, III, and IV were $47 \%, 55 \%, 69 \%$, and $82 \%$, indicating that ctDNA levels increase with cancer progression.

Regarding NSCLC, Guo et al. [130], investigated changes in ctDNA levels after surgical tumor resection in 41 NSCLC patients. They isolated ctDNA between 13 and 0 days before surgery and between 2 and 10 days after surgery, and the collected material was then compared with that isolated during surgery. Somatic driver mutations in tumor DNA (tDNA) and pre- and post-surgery plasma ctDNA sample pairs were identified by targeted sequencing in several genes including EGFR, KRAS, and TP53. The frequency of $91.7 \%$ of ctDNA mutations decreased after surgery. The agreement between ctDNA in plasma and tDNA was 78.1\%, and the test showed a sensitivity of $69.2 \%$ and a specificity of $93.3 \%$. Furthermore, the presence of ctDNA had a higher positive predictive value (94.7\%) than that of six tumor biomarkers in current clinical use.

In Abbosh et al. [131], the first 100 participants of TRACERx trials were enrolled, and their ctDNA was analysed using tumour-specific phylogenetic approach. They identified specific single nucleotide variants (SNVs) as independent predictors of ctDNA release. In particular, in patient CRUK0013, affected by early-stage lung ADC, ctDNA was detectable after surgery but not after adjuvant chemoradiation. ctDNA remained undetectable on surveillance and this correlated with long-term disease-free survival. The authors demonstrated that ctDNA can be cleared after effective adjuvant treatment, and that ctDNA clearance corresponds with improved patient survival. The use of an ultra-sensitive method in quantizing ctDNA levels has shown that its levels correlate with tumour volume measured at CT and PET in pre-treatment plasma. Patient P13 (stage IIB NSCLC) after RT presented at imaging a massive mass that could be interpreted as a residue of disease but at the same time the ctDNA levels were undetectable. The patient stayed free from disease for 22 months evidencing the validity of the result of ctDNA. In contrast, patient P14 (stage IIIB NSCLC) presented a complete response to radiological follow-up after chemoradiotherapy but increasing levels of ctDNA. Indeed, clinical progression was demonstrated 7 months later.

CTCs can be another marker of residual disease. In a recent Spanish study [132], CTCs were detected before and 1 month after surgery in a group of NSCLC patients (stage I-IIIA). The discovery of these cells after surgery was associated with a faster relapse. In addition, the preoperative standard uptake value (SUV) ${ }_{\max }$ value of the primary tumor was associated with postoperative CTCs presence.

As shown by the aforementioned studies, ctDNA can be used as a predictive marker for prognosis and treatment response. Their use in the evaluation of MRD could allow a greater customization of treatment. Adjuvant or salvage RT could be used in patients with increasing levels of ctDNA in the absence of clinically or radiologically evident residues while observation alone may be sufficient in patients with undetectable ctDNA levels. This approach would certainly improve the toxicity profile. ctDNA analysis may enable detection or therapy-resistant or dormant clones. It is currently impossible to obtain all this information, but ctDNA analysis may allow this in the not too distant future.

\section{Conclusions}

Liquid biopsy is a minimally invasive method to monitor lung cancer dynamics and has a promising role in RT with an amazing impact on daily practice. Its possible uses are numerous, from early diagnosis, to guiding therapy and evaluation of residual disease.

The identification of radiosensitivity and radioresistance markers would allow a better selection of patients to undergo RT; serial monitoring of ctDNA during treatment could identify early non-responders and thus allow adjustment to the radiation dose (i.e. dose escalation or de-escalation). All this will lead to increasingly personalized therapy for individual patients. Liquid biopsy also has a role in follow-up because some biomarkers can reveal recurrence of disease well before traditional imaging techniques.

To date, the main indication to the use of the liquid biopsy is the identification of mutations to guide targeted therapy in patients without macroscopic disease, as approved by the European Medicines Agency 
and the FDA. Many potential applications of liquid biopsy remain unexplored and numerous studies will be needed to prove the effectiveness of this method and its inclusion in the management of the NSCLC. Despite these limitations, if the current evidence is confirmed, liquid biopsy will significantly change the therapeutic possibilities and therefore the prognosis of NSCLC patients especially in the RT field.

\section{Abbreviations}

ADC: adenocarcinoma

$A L K$ : anaplastic lymphoma kinase

CEA: carcinoembryonic antigen

cfDNA: cell-free DNA

COPD: chronic obstructive pulmonary disease

CTCs: circulating tumor cells

ctDNA: circulating tumor DNA

ctRNA: circulating tumor RNA

ddPCR: droplet digital polymerase chain reaction

ILD: interstitial lung disease

IR: ionizing radiation

KEAP1: kelch-like ECH-associated protein 1

KRAS: Kirsten rat sarcoma viral oncogene homolog

LDCT: low-dose CT

MRD: minimum residual disease

NGS: next generation sequencing

NRF2: Nuclear factor erythroid 2-related factor 2

NSCLC: non-small cell lung cancer

NSE: neuron-specific enolase

PCR: polymerase chain reaction

PFS: progression free survival

qPCR: quantitative polymerase chain reaction

ROS: reactive oxygen species

RT: radiotherapy

SCLC: small cell lung cancer

SNPs: single nucleotide polymorphisms

SqCC: squamous cell carcinoma

TGF- $\beta$ : transforming growth factor $\beta$

TKIs: tyrosine kinase inhibitors

SBRT: stereotactic body irradiation

tDNA: tumor DNA

\section{Declarations}

\section{Author contributions}

AP and IRS contributed conception and design of the manuscript. AP, IRS, MVAO, JR, RMM, AR and MT wrote sections of the manuscript. All authors contributed to manuscript revision, read and approved the submitted version. 


\section{Conflict of interest}

The authors declare that they have no conflicts of interest.

\section{Ethical approval}

Not applicable.

\section{Consent to participate}

Not applicable.

\section{Consent to publication}

Not applicable.

\section{Availability of data and materials}

Not applicable.

\section{Funding}

Not applicable.

\section{Copyright}

(C) The Author(s) 2021.

\section{References}

1. Bray F, Ferlay J, Soerjomataram I, Siegel RL, Torre LA, Jemal A. (2018) Global cancer statistics 2018: GLOBOCAN estimates of incidence and mortality worldwide for 36 cancers in 185 countries. CA Cancer J Clin. 2018;68:394-424.

2. Travis WD, Brambilla E, Burke AP, Marx A, Nicholson AG. WHO classification of tumours of the lung, pleura, thymus and heart. 4th edition. Lyon: International Agency for Research on Cancer; 2015.

3. Overman MJ, Modak J, Kopetz S, Murthy R, Yao JC, Hicks ME, et al. Use of research biopsies in clinical trials: are risks and benefits adequately discussed? J Clin Oncol. 2013;31:17-22.

4. Coghlin CL, Smith LJ, Bakar S, Stewart KN, Devereux GS, Nicolson MC, et al. Quantitative analysis of tumor in bronchial biopsy specimens. J Thorac Oncol. 2010;5:448-52.

5. Gerlinger M, Rowan AJ, Horswell S, Math M, Larkin J, Endesfelder D, et al. Intratumor heterogeneity and branched evolution revealed by multiregion sequencing. N Engl J Med. 2012;366:883-92.

6. Diaz LA Jr, Bardelli A. Liquid biopsies: genotyping circulating tumor DNA. J Clin Oncol. 2014;32:579-86.

7. Mandel P, Metais P. Nuclear acids in human blood plasma. C R Seances Soc Biol Fil. 1948;142: 241-3. French.

8. Fleischhacker M, Schmidt B. Circulating nucleic acids (CNAs) and cancer--a survey. Biochim Biophys Acta. 2007;1775:181-232.

9. Newman AM, Bratman SV, To J, Wynne JF, Eclov NC, Modlin LA, et al. An ultrasensitive method for quantitating circulating tumor DNA with broad patient coverage. Nat Med. 2014;20:548-54.

10. Kustanovich A, Schwartz R, Peretz T, Grinshpun A. Life and death of circulating cell-free DNA. Cancer Biol Ther. 2019;20:1057-67.

11. Cheng F, Su L, Qian C. Circulating tumor DNA: a promising biomarker in the liquid biopsy of cancer. Oncotarget. 2016;7:48832-41.

12. Jiang $\mathrm{P}$, Chan $\mathrm{CW}$, Chan $\mathrm{KC}$, Cheng $\mathrm{SH}$, Wong J, Wong VW, et al. Lengthening and shortening of plasma DNA in hepatocellular carcinoma patients. Proc Natl Acad Sci USA. 2015;112:E1317-25. 
13. Madhavan D, Wallwiener M, Bents K, Zucknick M, Nees J, Schott S, et al. Plasma DNA integrity as a biomarker for primary and metastatic breast cancer and potential marker for early diagnosis. Breast Cancer Res Treat. 2014;146:163-74.

14. Chan KC, Jiang P, Zheng YW, Liao GJ, Sun H, Wong J, et al. Cancer genome scanning in plasma: detection of tumor-associated copy number aberrations single-nucleotide variants and tumoral heterogeneity by massively parallel sequencing. Clin Chem. 2013;59:211-24.

15. Wong IH, Lo YM, Zhang J, Liew CT, Ng MH, Wong N, et al. Detection of aberrant p16 methylation in the plasma and serum of liver cancer patients. Cancer Res. 1999;59:71-3.

16. Lee TH, Montalvo L, Chrebtow V, Busch MP. Quantitation of genomic DNA in plasma and serum samples: higher concentrations of genomic DNA found in serum than in plasma. Transfusion. 2001;41:276-82.

17. Normanno N, Denis MG, Thress KS, Ratcliffe M, Reck M. Guide to detecting epidermal growth factor receptor (EGFR) mutations in ctDNA of patients with advanced non-smallcell lung cancer. Oncotarget. 2017;8:12501-16.

18. Toro PV, Erlanger B, Beaver JA, Cochran RL,VanDenBerg DA, Yakim E, et al. Comparison of cell stabilizing blood collection tubes for circulating plasma tumor DNA. Clin Biochem.2015;48:993-8.

19. Schmidt B, Reinicke D, Reindl I, Bork I, Wollschläger B, Lambrecht N, et al. Liquid biopsy- performance of the PAXgene $囚$ blood ccfDNA tubes for the isolation and characterization of cell-free plasma DNA from tumor patients. Clin Chim Acta. 2017;469:94-8.

20. Merker JD, Oxnard GR, Compton C, Diehn M, Hurley P, Lazar AJ, et al. Circulating tumor DNA analysis in patients with cancer: American Society of Clinical Oncology and College of American Pathologist Joint Review. J Clin Oncol. 2018;36:1631-41.

21. Oxnard GR, Paweletz CP, Sholl LM. Genomic analysis of plasma cell-free DNA in patients with cancer. JAMA Oncol. 2017;3:740-1.

22. Wu KL, Tsai YM, Lien CT, Kuo PL, Hung AJ. The roles of microRNA in lung cancer. Int J Mol Sci. 2019;20:1611.

23. Jin X, Chen Y, Chen H, Fei S, Chen D, Cai X, et al. Evaluation of tumor-derived exosomal miRNA as potential diagnostic biomarkers for early-stage non-small cell lung cancer using next-generation sequencing. Clin Cancer Res. 2017;23:5311-9.

24. Liu Q, Yu Z, Yuan S, Xie W, Li C, Hu Z, et al. Circulating exosomal microRNAs as prognostic biomarkers for non-small-cell lung cancer. Oncotarget. 2017;8:13048-58.

25. Ashworth TR. A case of cancer in which cells similar to those in the tumours were seen in the blood after death. Med J Aust. 1869;14:146-7.

26. Micalizzi DS, Maheswaran S, Haber DA. A conduit to metastasis: circulating tumor cell biology. Genes Dev. 2017;31:1827-40.

27. Chaffer CL, Weinberg RA. A perspective on cancer cell metastasis. Science. 2011;331:1559-64.

28. Giuliano M, Giordano A, Jackson S, De Giorgi U, Mego M, Cohen EN, et al. Circulating tumor cells as early predictors of metastatic spread in breast cancer patients with limited metastatic dissemination. Breast Cancer Res. 2014;16:440.

29. Goodman OB Jr, Symanowski JT, Loudyi A, Fink LM, Ward DC, Vogelzang NJ. Circulating tumor cells as a predictive biomarker in patients with hormone-sensitive prostate cancer. Clin Genitourin Cancer. 2011;9:31-8.

30. Wang D, Yang Y, Jin L, Wang J, Zhao X, Wu G, et al. Prognostic models based on postoperative circulating tumor cells can predict poor tumor recurrence-free survival in patients with stage II-III colorectal cancer. J Cancer. 2019;10:4552-63.

31. Zhang J, Chen K, Fan ZH. Circulating tumor cell isolation and analysis. Adv Clin Chem. 2016;75:1-31. 
32. Earhart CM, Hughes CE, Gaster RS, Ooi CC, Wilson RJ, Zhou LY, et al. Isolation and mutational analysis of circulating tumor cells from lung cancer patients with magnetic sifters and biochips. Lab Chip. 2014;14:78-88.

33. Shaffer DR, Leversha MA, Danila DC, Lin O, Gonzalez-Espinoza R, Gu B, et al. Circulating tumor cell analysis in patients with progressive castration-resistant prostate cancer. Clin Cancer Res. 2007;13:2023-9.

34. Tong X, Yang L, Lang JC, Zborowski M, Chalmers JJ. Application of immunomagnetic cell enrichment in combination with RT-PCR for the detection of rare circulating head and neck tumor cells in human peripheral blood. Cytometry B Clin Cytom. 2007;72:310-23.

35. Catelain C, Pailler E, Oulhen M, Faugeroux V, Pommier AL, Farace F. Detection of gene rearrangements in circulating tumor cells: examples of ALK-, ROS1-, RET-rear-rangements in non-small-cell lung cancer and ERG-rearrange-ments in prostate cancer. Adv Exp Med Biol. 2017;994:169-79.

36. Walters S, Maringe C, Coleman MP, Peake MD, Butler J, Young N, et al; ICBP Module 1 Working Group. Lung cancer survival and stage at diagnosis in Australia, Canada, Denmark, Norway, Sweden and the UK: a population-based study, 2004-2007. Thorax. 2013;68:551-64.

37. National Lung Screening Trial Research Team; Aberle DR, Adams AM, Berg CD, Black WC, Clapp JD, Fagerstrom RM, et al. Reduced lung-cancer mortality with low-dose computed tomographic screening. N Engl J Med. 2011;365:395-409.

38. Gartman EJ, Jankowich MD, Baptiste J, Nici L. Providence VA lung cancer screening program: performance: comparison of local false positive and invasive procedure rates to published trial data. Am J Respir Crit Care Med. 2020;201:A2477.

39. Yousaf-Khan U, van der Aalst C, de Jong PA, Heuvelmans M, Scholten E, Lammers JW, et al. Final screening round of the NELSON lung cancer screening trial: the effect of a 2.5 -year screening interval. Thorax. 2017;72:48-56.

40. Newman AM, Bratman SV, To J, Wynne JF, Eclov NC, Modlin LA, et al. An ultrasensitive method for quantitating circulating tumor DNA with broad patient coverage. Nat Med. 2014;20:548-54.

41. Cohen JD, Li L, Wang Y, Thoburn C, Afsari B, Danilova L, et al. Detection and localization of surgically resectable cancers with a multi-analyte blood test. Science. 2018;359:926-30.

42. Zhang Y, Wang R, Song H, Huang G, Yi J, Zheng Y, et al. Methylation of multiple genes as a candidate biomarker in non-small cell lung cancer. Cancer Lett. 2011;303:21-8.

43. Wielscher M, Vierlinger K, Kegler U, Ziesche R, Gsur A, Weinhäusel A. Diagnostic performance of plasma DNA methylation profiles in lung cancer, pulmonary fibrosis and COPD. EBioMedicine. 2015;2:929-36.

44. Hulbert A, Jusue-Torres I, Stark A, Chen C, Rodgers K, Lee B, et al. Early detection of lung cancer using DNA promoter hypermethylation in plasma and sputum. Clin Cancer Res. 2016;23:1998-2005.

45. Liang $\mathrm{W}$, Zhao $\mathrm{Y}$, Huang $\mathrm{W}$, Gao Y, Xu W, Tao J, et al. Non-invasive diagnosis of early-stagelung cancer using high-throughput targeted DNA methylation sequencing of circulating tumor DNA (ctDNA). Theranostics. 2019;9:2056-70.

46. Krebs MG, Hou JM, Sloane R, Lancashire L, Priest L, Nonaka D, et al. Analysis of circulating tumor cells in patients with non-small cell lung cancer using epithelial marker-dependent and -independent approaches. J Thorac Oncol. 2012;7:306-15.

47. Hofman V, Ilie MI, Long E, Selva E, Bonnetaud C, Molina T, et al. Detection of circulating tumor cells as a prognostic factor in patients undergoing radical surgery for non-small-cell lung carcinoma: comparison of the efficacy of the CellSearch Assay ${ }^{\mathrm{TM}}$ and the isolation by size of epithelial tumor cell method. Int J Cancer. 2011;129:1651-660.

48. Ilie M, Hofman V, Long-Mira E, Selva E, Vignaud JM, Padovani B, et al. "Sentinel" circulating tumor cells allow early diagnosis of lung cancer in patients with chronic obstructive pulmonary disease. PLoS One. 2014;9:e111597. 
49. Yu Y, Chen Z, Dong J, Wei P, Hu R, Zhou C, et al. Folate receptor-positive circulating tumor cells as a novel diagnostic biomarker in non-small cell lung cancer. Transl Oncol. 2013;6:697-702.

50. Ma J, Lin Y, Zhan M, Mann DL, Stass SA, Jiang F. Differential miRNA expressions in peripheral blood mononuclear cells for diagnosis of lung cancer. Lab Invest. 2015;95:1197-206.

51. Nadal E, Truini A, Nakata A, Lin J, Reddy RM, Chang AC, et al. A novel serum 4-microRNA signature for lung cancer detection. Sci Rep. 2015;5:12464.

52. Dou H, Wang Y, Su G, Zhao S. Decreased plasma let-7c and miR-152 as noninvasive biomarker for nonsmall-cell lung cancer. Int J Clin Exp Med. 2015;8:9291-8.

53. Tandberg DJ, Tong BC, Ackerson BG, Kelsey CR. Surgery versus stereotactic body radiation therapy for stage I non-small cell lung cancer: a comprehensive review. Cancer. 2018;124:667-78.

54. Lacombe J, Azria D, Mange A, Solassol J. Proteomic approaches to identify biomarkers predictive of radiotherapy outcomes. Expert Rev Proteomics. 2013;10:33-42

55. Baumann M, Krause M, Overgaard J, Debus J, Bentzen SM, Daartz J, et al. Radiation oncology in the era of precision medicine. Nat Rev Cancer. 2016;16:234-49.

56. Barker HE, Paget JT, Khan AA, Harrington KJ. The tumour microenvironment after radiotherapy: mechanisms of resistance and recurrence. Nat Rev Cancer. 2015;15:409-25.

57. Dagogo-Jack I, Shaw AT. Tumour heterogeneity and resistance to cancer therapies. Nat Rev Clin Oncol. 2018;15:81-94.

58. Li W, Qiu T, Guo L, Ling Y, Gao Y, Ying J, et al. Primary and acquired EGFR T790M-mutant NSCLC patients identified by routine mutation testing show different characteristics but may both respond to osimertinib treatment. Cancer Lett. 2018;423:9-15.

59. Zhang Y, Lin Q, Xu T, Deng W, Yu J, Liao Z, et al. Out of the darkness and into the light: new strategies for improving treatments for locally advanced non-small cell lung cancer. Cancer Lett. 2018;421:59-62.

60. Azria D, Lapierre A, Gourgou S, De Ruysscher D, Colinge J, Lambin P, et al. Data-based radiation oncology: design of clinical trials in the toxicity biomarkers era. Front Oncol. 2017;7:83.

61. Bibault JE, Fumagalli I, Ferté C, Chargari C, Soria JC, Deutsch E. Personalized radiation therapy and biomarker-driven treatment strategies: a systematic review. Cancer Metastasis Rev. 2013;32:479-92.

62. Schwarzenbach H, Pantel K. Circulating DNA as biomarker in breast cancer. Breast Cancer Res. 2015;17:136.

63. Hocking J, Mithraprabhu S, Kalff A, Spencer A. Liquid biopsies for liquid tumors: emerging potential of circulating free nucleic acid evaluation for the management of hematologic malignancies. Cancer Biol Med. 2016;13:215-25.

64. Okajima W, Komatsu S, Ichikawa D, Miyamae M, Ohashi T, Imamura T, et al. Liquid biopsy in patients with hepatocellular carcinoma: circulating tumor cells and cell-free nucleic acids. World J Gastroenterol. 2017;23:5650-68.

65. Calapre L, Warburton L, Milward M, Ziman M, Gray ES. Circulating tumour DNA (ctDNA) as a liquid biopsy for melanoma. Cancer Lett. 2017;404:62-9.

66. Duldulao MP, Lee W, Nelson RA, Li W, Chen Z, Kim J, et al. Mutations in specific codons of the KRAS oncogene are associated with variable resistance to neoadjuvant chemoradiation therapy in patients with rectal adenocarcinoma. Ann Surg Oncol. 2013;20:2166-71.

67. Jeong Y, Hoang NT, Lovejoy A, Stehr H, Newman AM, Gentles AJ, et al. Role of KEAP1/NRF2 and TP53 mutations in lung squamous cell carcinoma development and radiation resistance. Cancer Discov. 2017;7:86-101.

68. Cancer Genome Atlas Research Network. Comprehensive molecular characterization of urothelial bladder carcinoma. Nature. 2014;507:315-22. 
69. Ojesina AI, Lichtenstein L, Freeman SS, Pedamallu CS, Imaz-Rosshandler I, Pugh TJ, et al. Landscape of genomic alterations in cervical carcinomas. Nature. 2014;506:371-5.

70. Gao YB, Chen ZL, Li JG, Hu XD, Shi XJ, Sun ZM, et al. Genetic landscape of esophageal squamous cell carcinoma. Nat Genet. 2014;46:1097-102.

71. Cancer Genome Atlas Research Network. Comprehensive genomic characterization of head and neck squamous cell carcinomas. Nature. 2015;517:576-82.

72. Yard BD, Adams DJ, Chie EK, Tamayo P, Battaglia JS, Gopal P, et al. A genetic basis for the variation in the vulnerability of cancer to DNA damage. Nat Commun. 2016;7:11428.

73. Satoh H, Moriguchi T, Takai J, Ebina M, Yamamoto M. Nrf2 prevents initiation but accelerates progression through the Kras signaling pathway during lung carcinogenesis. Cancer Res. 2013;73:4158-68.

74. Le Gal K, Ibrahim MX, Wiel C, Sayin VI, Akula MK, Karlsson C, et al. Antioxidants can increase melanoma metastasis in mice. Sci Transl Med. 2015;7:308re8.

75. Itoh K, Wakabayashi N, Katoh Y, Ishii T, Igarashi K, Engel JD, et al. Keap1 represses nuclear activation of antioxidant responsive elements by Nrf2 through binding to the amino-terminal Neh2 domain. Genes Dev. 1999;13:76-86.

76. Kobayashi A, Kang MI, Okawa H, Ohtsuji M, Zenke Y, Chiba T, et al. Oxidative stress sensor Keap1 functions as an adaptor for Cul3-based E3 ligase to regulate proteasomal degradation of Nrf2. Mol Cell Biol. 2004;24:7130-9.

77. Hall EJ, Giaccia AJ. Radiobiology for the Radiologist 7th edition. Philadelphia: Lippincottt Williams \& Wilkins; 2011.

78. Porcellini E, Laprovitera N, Riefolo M, Ravaioli M, Garajova I, Ferracin M. Epigenetic and epitranscriptomic changes in colorectal cancer: diagnostic, prognostic, and treatment implications. Cancer Lett. 2018;419:84-95.

79. Pinson H, Hallaert G, Van der Meulen J, Dedeurwaerdere F, Vanhauwaert D, Van den Broecke C, et al. Weak MGMT gene promoter methylation confers a clinically significant survival benefit in patients with newly diagnosed glioblastoma: a retrospective cohort study. J Neurooncol. 2020;146:55-62.

80. Fischer JR, Ohnmacht U, Rieger N, Zemaitis M, Stoffregen C, Kostrzewa M, et al. Promoter methylation of RASSF1A, RAR $\beta$ and DAPK predict poor prognosis of patients with malignant mesothelioma. Lung Cancer. 2006;54:109-16.

81. Haldrup C, Mundbjerg K, Vestergaard EM, Lamy P, Wild P, Schulz WA, et al. DNA methylation signatures for prediction of biochemical recurrence after radical prostatectomy of clinically localized prostate cancer. J Clin Oncol. 2013;31:3250-8.

82. Chung HH, Kuo CC, Hsiao CW, Chen CY, Hu JM, Hsu CH, et al. A novel prognostic DNA methylation panel for colorectal cancer. Int J Mol Sci. 2019;20:4672.

83. Rodríguez-Paredes M, Esteller M. Cancer epigenetics reaches mainstream oncology. Nat Med. 2011; 17:330-9.

84. Thienpont B, Steinbacher J, Zhao H, D'Anna F, Kuchnio A, Ploumakis A, et al. Tumour hypoxia causes DNA hypermethylation by reducing TET activity. Nature. 2016;537:63-8.

85. Chai Y, Lam RK, Calaf GM, Zhou H, Amundson S, Hei TK. Radiation-induced non-targeted response in vivo: role of the TGFbeta-TGFBR1-COX-2 signalling pathway. Br J Cancer. 2013;108:1106-12.

86. Anscher MS. Targeting the TGF-beta1 pathway to prevent normal tissue injury after cancer therapy. Oncologist. 2010;15:350-9.

87. Ehrhart EJ, Segarini P, Tsang ML, Carroll AG, Barcellos-Hoff MH. Latent transforming growth factor beta1 activation in situ: quantitative and functional evidence after low dose gamma-irradiation. FASEB J. 1997;11:991-1002. 
88. Rube CE, Uthe D, Schmid KW, Richter KD, Wessel J, Schuck A, et al. Dose-dependent induction of transforming growth factor beta (TGF-beta) in the lung tissue of fibrosis-prone mice after thoracic irradiation. Int J Radiat Oncol Biol Phys. 2000;47:1033-42.

89. Anscher MS, Thrasher B, Rabbani Z, Teicher B, Vujaskovic Z. Antitransforming growth factor-beta antibody 1D11 ameliorates normal tissue damage caused by high-dose radiation. Int J Radiat Oncol Biol Phys. 2006;65:876-81.

90. Bouquet F, Pal A, Pilones KA, Demaria S, Hann B, Akhurst RJ, et al. TGF beta 1 inhibition increases the radiosensitivity of breast cancer cells in vitro and promotes tumor control by radiation in vivo. Clin Cancer Res. 2011;17:6754-65.

91. Hardee ME, Marciscano AE, Medina-Ramirez CM, Zagzag D, Narayana A, Lonning SM, et al. Resistance of glioblastoma-initiating cells to radiation mediated by the tumor microenvironment can be abolished by inhibiting transforming growth factor- $\beta$. Cancer Res. 2012;72:4119-29.

92. Akimoto T, Hunter NR, Buchmiller L, Mason K, Ang KK, Milas L. Inverse relationship between epidermal growth factor receptor expression and radiocurability of murine carcinomas. Clin Cancer Res. 1999;5:2884-90.

93. Bernhard EJ, Stanbridge EJ, Gupta S, Gupta AK, Soto D, Bakanauskas VJ, et al. Direct evidence for the contribution of activated $\mathrm{N}$-ras and $\mathrm{K}$-ras oncogenes to increased intrinsic radiation resistance in human tumor cell lines. Cancer Res. 2000;60:6597-600.

94. Diehl F, Schmidt K, Choti MA, Romans K, Goodman S, Li M, et al. Circulating mutant DNA to assess tumor dynamics. Nat Med. 2008;14:985-90.

95. Yao W, Mei C, Nan X, Hui L. Evaluation and comparison of in vitro degradation kinetics of DNA in serum, urine and saliva: a qualitative study. Gene. 2016;590:142-8.

96. Lo YM, Chan LY, Lo KW, Leung SF, Zhang J, Chan AT, et al. Quantitative analysis of cell-free Epstein-Barr virus DNA in plasma of patients with nasopharyngeal carcinoma. Cancer Res. 1999;59:1188-91.

97. Rago C, Huso DL, Diehl F, Karim B, Liu G, Papadopoulos N, et al. Serial assessment of human tumor burdens in mice by the analysis of circulating DNA. Cancer Res. 2007;67:9364-70.

98. Lo YM, Leung SF, Chan LY, Chan AT, Lo KW, Johnson PJ, et al. Kinetics of plasma Epstein-Barr virus DNA during radiation therapy for nasopharyngeal carcinoma. Cancer Res. 2000;60:2351-5.

99. Osumi H, Shinozaki E, Yamaguchi K, Zembutsu H. Clinical utility of circulating tumor DNA for colorectal cancer. Cancer Sci. 2019;110:1148-55.

100. Bentzen SM, Overgaard J. Patient-to-patient variability in the expression of radiation-induced normal tissue injury. Semin Radiat Oncol. 1994;4:68-80.

101. Kozin SV, Niemierko A, Huang P, Silva J, Doppke KP, Suit HD. Inter- and intramouse heterogeneity of radiation response for a growing paired organ. Radiat Res. 2008;170:264-7.

102. Krause M, Gurtner K, Deuse Y, Baumann M. Heterogeneity of tumour response to combined radiotherapy and EGFR inhibitors: differences between antibodies and TK inhibitors. Int J Radiat Biol. 2009;85:943-54.

103. Meacham CE, Morrison SJ. Tumour heterogeneity and cancer cell plasticity. Nature. 2013;501:328-37.

104. Jamal-Hanjani M, Wilson GA, McGranahan N, Birkbak NJ, Watkins TBK, Veeriah S, et al; TRACERx Consortium. Tracking the evolution of non small-cell lung cancer. N Engl J Med. 2017;376:2109-21.

105. Renovanz M, Kim EL. Intratumoral heterogeneity, its contribution to therapy resistance and methodological caveats to assessment. Front Oncol. 2014;4:142.

106. Rocco JW. Mutant allele tumor heterogeneity (MATH) and head and neck squamous cell carcinoma. Head Neck Pathol. 2015;9:1-5.

107. Morrison R, Schleicher SM, Sun Y, Niermann KJ, Kim S, Spratt DE, et al. Targeting the mechanisms of resistance to chemotherapy and radiotherapy with the cancer stem cell hypothesis. J Oncol. 2011;2011:941876. 
108. Kerns SL, Ostrer H, Rosenstein BS. Radiogenomics: using genetics to identify cancer patients at risk for development of adverse effects following radiotherapy. Cancer Discov. 2014;4:155-65.

109. Ho AY, Atencio DP, Peters S, Stock RG, Formenti SC, Cesaretti JA, et al. Genetic predictors of adverse radiotherapy effects: the Gene-PARE project. Int J Radiat Oncol Biol Phys. 2006;65:646-55.

110. Azria D, Ozsahin M, Kramar A, Peters S, Atencio DP, Crompton NE, et al. Single nucleotide polymorphisms, apoptosis, and the development of severe late adverse effects after radiotherapy. Clin Cancer Res. 2008;14:6284-8.

111. Gaffney DK, Brohet RM, Lewis CM, Holden JA, Buys SS, Neuhausen SL, et al. Response to radiation therapy and prognosis in breast cancer patients with BRCA1 and BRCA2 mutations. Radiother Oncol. 1998;47:129-36.

112. Cesaretti JA, Stock RG, Atencio DP, Peters SA, Peters CA, Burri RJ, et al. A genetically determined dosevolume histogram predicts for rectal bleeding among patients treated with prostate brachytherapy. Int J Radiat Oncol Biol Phys. 2007;68:1410-6.

113. Tucker SL, Li M, Xu T, Gomez D, Yuan X, Yu J, et al. Incorporating single-nucleotide polymorphisms into the Lyman model to improve prediction of radiation pneumonitis. Int J Radiat Oncol Biol Phys. 2013;85:251-7.

114. Kerns SL, Ostrer H, Stock R, Li W, Moore J, Pearlman A, et al. Genome wide association study to identify single nucleotide polymorphisms (SNPs) associated with the development of erectile dysfunction in African-American men following radiotherapy for prostate cancer. Int J Radiat Oncol Biol Phys. 2010;78:1292-300.

115. Lynch TJ, Bell DW, Sordella R, Gurubhagavatula S, Okimoto RA, Brannigan BW, et al. Activating mutations in the epidermal growth factor receptor underlying responsiveness of non-small-cell lung cancer to gefitinib. N Engl J Med. 2004;350:2129-39.

116. Chong CR, Jänne PA. The quest to overcome resistance to EGFR-targeted therapies in cancer. Nat Med. 2013;19:1389-400.

117. Mayo-de-Las-Casas C, Jordana-Ariza N, Garzón-Ibañez M, Balada-Bel A, Bertrán-Alamillo J, ViteriRamírez S, et al. Large scale, prospective screening of EGFR mutations in the blood of advanced NSCLC patients to guide treatment decisions. Ann Oncol. 2017;28:2248-55.

118. Buder A, Hochmair MJ, Schwab S, Bundalo T, Schenk P, Errhalt P, et al. Cell-free plasma DNA-guided treatment with osimertinib in patients with advanced EGFR-mutated NSCLC. J Thorac Oncol. 2018;13:821-30.

119. Provencio M, Torrente M, Calvo V, Gutiérrez L, Pérez-Callejo D, Pérez-Barrios C, et al. Dynamic circulating tumor DNA quantificaton for the individualization of non-small-cell lung cancer patients treatment. Oncotarget. 2017;8:60291-8.

120. Ni J, Weng L, Liu Y, Sun Z, Bai C, Wang Y. Dynamic monitoring of EGFR mutations in circulating cellfree DNA for EGFR-mutant metastatic patients with lung cancer: early detection of drug resistance and prognostic significance. Oncol Lett. 2017;13:4549-57.

121. Takahashi T, Sonobe M, Kobayashi M, Yoshizawa A, Menju T, Nakayama E, et al. Clinicopathologic features of non-small-cell lung cancer with EML4-ALK fusion gene. Ann Surg Oncol. 2010;17:889-97.

122. Gainor JF, Dardaei L, Yoda S, Friboulet L, Leshchiner I, Katayama R, et al. Molecular mechanisms of resistance to first- and second-generation ALK inhibitors in ALK-rearranged lung cancer. Cancer Discov. 2016;6:1118-33.

123. Katayama R, Friboulet L, Koike S, Lockerman EL, Khan TM, Gainor JF, et al. Two novel ALK mutations mediate acquired resistance to the next-generation ALK inhibitor alectinib. Clin Cancer Res. 2014;20:5686-96.

124. Shaw AT, Ou SH, Bang YJ, Camidge DR, Solomon BJ, Salgia R, et al. Crizotinib in ROS1 -rearranged nonsmall-cell lung cancer. N Engl J Med. 2014;371:1963-71. 
125. Drilon A, Rekhtman N, Arcila M, Wang L, Ni A, Albano M, et al. Cabozantinib in patients with advanced RET-rearranged non-small-cell lung cancer: an open-label, single-centre, phase 2, single-arm trial. Lancet Oncol. 2016;17:1653-60.

126. Pan W, Yang Y, Zhu H, Zhang Y, Zhou R, Sun X. KRAS mutation is a weak, but valid predictor for poor prognosis and treatment outcomes in NSCLC: a meta-analysis of 41 studies. Oncotarget. 2016;7:8373-88.

127. Janning M, Kobus F, Babayan A, Wikman H, Velthaus JL, Bergmann S, et al. Determination of PD-L1 expression in circulating tumor cells of NSCLC patients and correlation with response to PD-1/PD-L1 inhibitors. Cancers (Basel). 2019;11:835.

128. Diehl F, Schmidt K, Choti MA, Romans K, Goodman S, Li M, et al. Circulating mutant DNA to assess tumor dynamics. Nat Med. 2008;14:985-90.

129. Bettegowda C, Sausen M, Leary RJ, Kinde I, Wang Y, Agrawal N, et al. Detection of circulating tumor DNA in early- and late-stage human malignancies. Sci Transl Med. 2014;6:224ra24.

130. Guo N, Lou F, Ma Y, Li J, Yang B, Chen W, et al. Circulating tumor DNA detection in lung cancer patients before and after surgery. Sci Rep. 2016;6:33519.

131. Abbosh C, Birkbak NJ, Wilson GA, Jamal-Hanjani M, Constantin T, Salari R, et al. Phylogenetic ctDNA analysis depicts early-stage lung cancer evolution. Nature. 2017;545:446-51. Erratum in: Nature. 2018;554:264.

132. Bayarri-Lara CI, de Miguel Pérez D, Cueto Ladrón de Guevara A, Rodriguez Fernández A, Puche JL, Sánchez-Palencia Ramos A, et al. Association of circulating tumour cells with early relapse and 18F-fluorodeoxyglucose positron emission tomography uptake in resected non-small-cell lung cancers. Eur J Cardiothorac Surg. 2017;52:55-62. 